\title{
Investigation of local electrical properties of coincidence-site-lattice boundaries in location-controlled silicon islands using scanning capacitance microscopy
}

\author{
Nobuyuki Matsuki, ${ }^{a}$ Ryoichi Ishihara, Alessando Baiano, and Kees Beenakker \\ Faculty of Electronic Components, Technology and Materials (ECTM), Delft Institute of Microsystems and \\ Nanoelectronics (DIMES), Delft University of Technology, Feldmannweg 17, P.O. Box 5053, \\ 2600 GB Delft, The Netherlands
}

(Received 28 February 2008; accepted 11 July 2008; published online 11 August 2008)

\begin{abstract}
We used scanning capacitance microscopy (SCM) to investigate the electrical activity of grain boundaries consisting of random and coincidence-site-lattice (CSL) boundaries in location-controlled silicon islands, which were fabricated using the $\mu$-Czochralski process with an excimer laser. The SCM results suggest that the electrical activity of the $\{122\} \sum 9$ CSL boundary is much smaller than that of a random boundary, and the $\{111\} \sum 3$ CSL boundary is negligible. This is consistent with previous theoretical predictions and experimental results for thin-film transistors. (C) 2008 American Institute of Physics. [DOI: 10.1063/1.2968663]
\end{abstract}

Low-temperature polycrystalline silicon (LTPS) thinfilm transistors (TFTs) have been used for liquid crystal display pixel and peripheral drivers. The typical mobility of commercially produced LTPS-TFTs is currently about $100 \mathrm{~cm}^{2} / \mathrm{V} \mathrm{s}$. This value is not high enough to manufacture central processing units with performance characteristics comparable to units based on bulk monocrystalline silicon. The lower mobility is attributed to the high density of electrical barriers induced by random boundaries (RBs) in the channel. To mitigate this effect, various types of LTPS have been proposed to enlarge the grains in polycrystalline silicon. ${ }^{1-8}$ We have developed an advanced LTPS method, designated the $\mu$-Czochralski ( $\mu$-Cz) process, which enables silicon islands to be produced at predetermined positions with a diameter of up to $9 \mu \mathrm{m} .{ }^{9}$ By eliminating almost all the RBs in the silicon islands, the TFTs fabricated inside the grain attained a field-effect mobility of $597 \mathrm{~cm}^{2} / \mathrm{V} \mathrm{s}{ }^{10}$ However, when coincidence-site-lattice (CSL) boundaries lie across the current flow direction, the mobility was shown to decrease by $10 \%$ on average. ${ }^{11}$ The $\{111\} \mathrm{Si} \Sigma 3$ and $\{122\} \mathrm{Si}$ $\Sigma 9$ are the CSL boundaries commonly found in polycrystalline silicon. ${ }^{12}$ Other $\Sigma 3^{n}$ CSL boundaries with a common rotation axis of $\langle 011\rangle$, such as $\{112\} \Sigma 3, \Sigma 9\{114\}$, and $\Sigma 9\{111\} /\{115\}$, have a lower probability of being found due to their thermal instability. ${ }^{12}$ Transmission electron microscopy revealed that the trend was the same in silicon islands fabricated by the $\mu$-Cz process. ${ }^{13}$

Experimental observations of the electrical activity of grain boundaries (GBs) have been performed mainly by electron beam induced current (EBIC) microscopy. ${ }^{14-16}$ Although the EBIC can provide quantitative information about the electrical activity of GBs, its resolution is limited by the carrier diffusion length, which is not small enough for the size of grains and the depletion width of GBs. Recent progress in scanning probe microscopy (SPM) allows various methods of electrical measurement to be applied at the nanoscale. ${ }^{17}$ The SPM is now being employed to investigate defects in semiconductor materials. Several groups have re-

${ }^{a)}$ Electronic mail: n.matsuki@tudelft.nl. ported the electrical properties of RBs using scanning probe techniques. ${ }^{18,19}$ More recently, the interfacial states and potential barriers of GBs were investigated using Kelvin probe microscopy. ${ }^{20}$ Using the computational semiempirical tightbinding method, Kohyama et al. ${ }^{21}$ predicted that the $\{122\} \mathrm{Si}$ $\Sigma 9$ CSL boundary has no states inside the minimum band gap between the valence-band maximum and the conduction band minimum; however, there are some degrees of localized states at valence and conduction band edges due to structural distortion induced by five-member and seven-member silicon rings inserted at the boundary. The difference in localized states can induce a change in capacitance at the CSL boundary. Here, we report the results of direct and systematic characterization of the electrical activity of random and CSL boundaries by scanning capacitance microscopy (SCM).

The sample used for SCM measurements was fabricated using a process that consisted of formation of grain filters, amorphous silicon, and laser crystallization. The details have been described elsewhere. ${ }^{22}$ The silicon layer was fixed at $250 \mathrm{~nm}$. The laser energy density was fixed at $1000 \mathrm{~mJ} / \mathrm{cm}^{2}$. Electron backscattered diffraction (EBSD) analysis was carried out to identify CSL boundaries in the location-controlled silicon grains. An orientation imaging microscopy system equipped with a scanning electron microscope (SEM) (Philips SEM 525M) was used for the analysis. SCM was performed using the multipurpose SPM NTEGRA (NTI-Europe

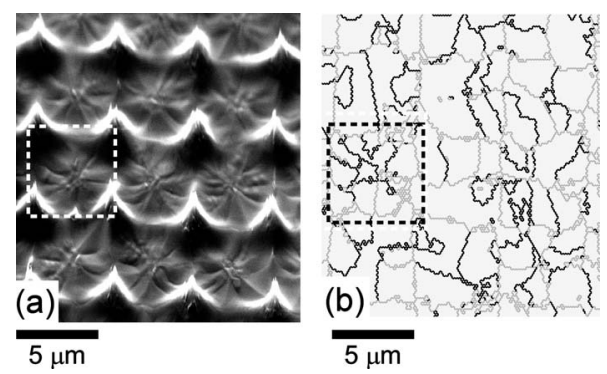

FIG. 1. (a) SEM image of part of the silicon islands fabricated by the $\mu-\mathrm{Cz}$ process. The dashed square corresponds to the area chosen for detailed observation in Fig. 2. (b) SCM image of the same $3 \times 3$ silicon islands. Black and gray lines indicate CSL and RBs, respectively. 


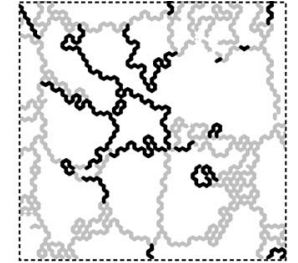

(a) $\Sigma 3(+\mathrm{RB})$
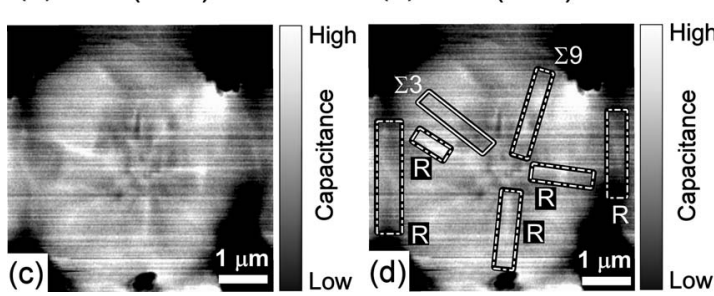

FIG. 2. EBSD and SCM results for the silicon island indicated with a dashed square in Fig. 1: (a) and (b) show the results of EBSD analysis of the CSL boundaries (black line) and RBs (gray lines) for $\Sigma 3$ and $\Sigma 9$, respectively. (c) shows the raw image from SCM. (d) shows the same SCM image as (c) but with lines to indicate the various regions. Solid line: $\Sigma 3$; dashed line: $\Sigma 9$; and dashed-dotted lines: random boundary (r).

Co., Ltd.). Platinum-coated silicon cantilevers were utilized during the measurements. The modulation frequency of the sample bias for the SCM was $10 \mathrm{MHz}$.

Figure 1(a) shows a SEM image of the locationcontrolled silicon grains obtained. Each silicon island has radiated striations initiating from the grain center, which is an evidence of the crystallization that propagated from the grain filter. The grains have a square-shaped configuration measuring approximately $5 \mu \mathrm{m}$ on one side. The size of the square shape suggests that the laser-annealed crystallization had the potential to extend the island beyond the size of $5 \mu \mathrm{m}$ if not for the collisions with adjacent grains. The results of EBSD analysis for the same region are shown in Fig. 1(b). The ratio of the $\Sigma 3$ boundary to the $\Sigma 9$ CSL boundary, which were included in nine grains consisting of a total area of $160 \mu \mathrm{m}^{2}$, was approximately $16: 3$, which is consistent with our previous results obtained by transmission electron microscopy. ${ }^{13}$ Figures 2(a) and 2(b) show the EBSD results for one of the silicon grains indicated with a white dashed square in Fig. 1. Figure 2(c) shows the SCM results for the same silicon island. The SCM image was obtained at a sample bias of $-2 \mathrm{~V}$. The brighter and darker colors in the SCM image correspond to regions of higher and lower capacitance, respectively. Specific regions corresponding to the CSL and RBs are indicated by rounded rectangles in Fig. 2(d). The $\Sigma 3$ boundary is indicated by a straight line, the $\Sigma 9$ boundary is indicated with a dotted line, and the random boundary is indicated with a dashed-dotted line. As shown in
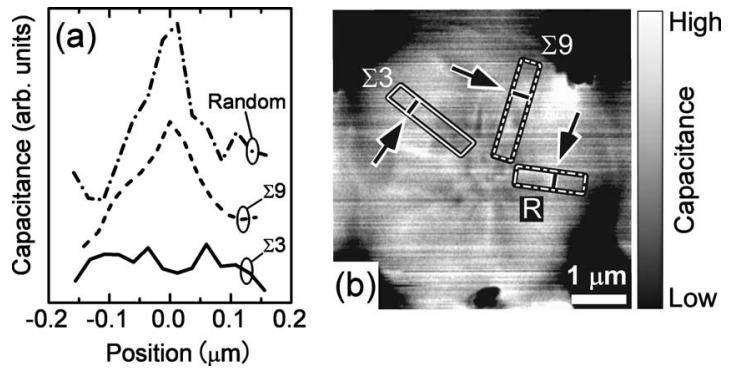

FIG. 3. (a) Cross-sectional profiles of capacitance at GBs. Positions of the cross sections are indicated in (b).

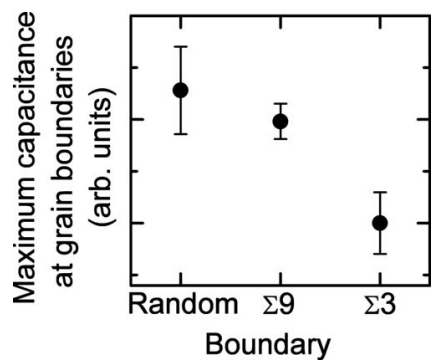

FIG. 4. Maximum capacitance of the cross-sectional profiles of capacitance at the CSL and random GBs.

Fig. 2(d), the evidence for the presence of excess capacitance is strongest at the RB. The $\{122\} \sum 9 \mathrm{CSL}$ boundaries exhibit excess capacitance to a lesser extent and the $\mathrm{Si}\{111\} \sum 3$ CSL boundaries do not exhibit any evidence of redundant capacitance. Figure 3(a) shows the cross-sectional profiles of the capacitance taken across each grain boundary. The crosssectional positions are indicated in Fig. 3(b). It can clearly be seen that the maximum capacitance (i.e., the peak height) in the cross-sectional profile differs between the $\Sigma 3$ and $\Sigma 9$ CSL boundaries and RBs. Figure 4 shows the results of analysis of the maximum capacitance for multiple crosssectional profiles extracted from the same silicon islands. The maximum capacitance plotted in Fig. 4 is derived by the peak height of the Gaussian function that was fitted to the experimental data of the cross-sectional profile. Comparing the maximum capacitance of the random $\left(C_{\mathrm{RB}}\right), \Sigma 3$, and $\Sigma 9$ boundaries, they are arranged in descending order as follows: $C_{\mathrm{RB}}>C_{\Sigma 9}>C_{\Sigma 3}$. A lower maximum capacitance suggests a higher density of carrier-trapping sites; representing the density as $D$, this can be described as $D_{\mathrm{RB}}>D_{\Sigma 9}>D_{\Sigma 3}$. This trend is consistent with the theoretical prediction that presumes the existence of localized states at the valence and conduction band edges. ${ }^{23}$ This result also provides further evidence for the observation that the CSL boundary does not influence the performance of TFT compared to the RBs. ${ }^{11}$

In summary, we performed direct characterization of $\{111\} \sum 3$ and $\{122\} \sum 9$ CSL boundaries using SCM. The electrical activities of the $\{111\} \sum 3$ and $\{122\} \sum 9$ CSL boundaries were negligible and much less than those found in RBs.

We would like to thank Dr. Y. Hiroshima, Dr. S. Inoue, and Dr. T. Hara of SEIKO EPSON Corporation for helpful discussions.

${ }^{1}$ H. Kuriyama, T. Kuwahara, S. Ishida, T. Nohda, K. Sano, H. Iwata, S. Noguchi, S. Kiyama, S. Tsuda, S. Nakano, S. Osumi, and Y. Kuwano, Jpn. J. Appl. Phys., Part 1 31, 4550 (1992).

${ }^{2}$ R. Ishihara and M. Matsumura, Electron. Lett. 31, 1956 (1995).

${ }^{3}$ S. W. Lee and S. K. Joo, IEEE Electron Device Lett. 17, 160 (1996).

${ }^{4}$ N. Higashi, G. Nalazawa, T. Asano, M. Miyasaka, and J. Stoemenos, Jpn. J. Appl. Phys., Part 1 45, 4347 (2006).

${ }^{5}$ J. S. Im and H. J. Kim, Appl. Phys. Lett. 64, 2303 (1994).

${ }^{6}$ M. Tai, M. Hatano, S. Yamaguchi, T. Noda, S. K. Park, T. Shiba, and M. Ohkura, IEEE Trans. Electron Devices 51, 934 (2004).

${ }^{7}$ A. Hara, F. Takeuchi, M. Takei, K. Suga, K. Yoshino, M. Chida, Y. Sano, and N. Sasaki, Jpn. J. Appl. Phys., Part 2 41, L311 (2002).

${ }^{8}$ C. H. Oh, M. Ozawa, and M. Matsumura, Jpn. J. Appl. Phys., Part 2 37, L492 (1998).

${ }^{9}$ R. Ishihara, P. C. van der Wilt, B. D. van Dijk, A. Burtsev, J. W. Metselaar, and C. I. M. Beenakker, Thin Solid Films 427, 77 (2003).

${ }^{10}$ R. Ishihara, Y. Hiroshima, D. Abe, B. D. van Dijk, P. C. van der Wilt, S. Higashi, S. Inoue, T. Shimoda, J. W. Metselaar, and C. I. M. Beenakker, IEEE Trans. Electron Devices 51, 500 (2004). 
${ }^{11}$ R. Ishihara, M. He, V. Rana, Y. Hiroshima, S. Inoue, T. Shimoda, J. W. Metselaar, and C. I. M. Beenakker, Thin Solid Films 487, 97 (2005).

${ }^{12}$ W. Garg, A. T. Clark, and J. P. Hirth, Philos. Mag. A 59, 479 (1989).

${ }^{13}$ R. Ishihara, D. Danciu, F. Tichelaar, M. He, Y. Hiroshima, S. Inoue, T. Shimoda, J. W. Metselaar, and C. I. M. Beenakker, J. Cryst. Growth 299, 316 (2007).

${ }^{14}$ Z.-J. Wang, S. Tsurekawa, K. Ikeda, T. Sekiguchi, and T. Watanabe, Interface Sci. 7, 197 (1999).

${ }^{15}$ J. Chen, T. Sekiguchi, D. Yang, F. Yin, K. Kido, and S. Tsurekwa, J. Appl. Phys. 96, 5491 (2004)

${ }^{16}$ J. Chen, T. Sekiguchi, R. Xie, P. Ahmet, T. Chikyo, D. Yang, S. Ito, and F. Yin, Scr. Mater. 52, 1211 (2005).

${ }^{17}$ Scanning Probe Microscopy-Electrical and Electrochemical Phenomena at the Nanoscale, edited by S. Kalinin and A. Gruverman (Springer, New York, 2007).

${ }^{18}$ I. Visoly-Fisher, S. R. Cohen, K. Gartsman, A. Ruzin, and D. Cahen, Adv. Funct. Mater. 16, 649 (2006)

${ }^{19}$ H. Yamagiwa, S. Abo, F. Wakaya, M. Takai, T. Sakamoto, H. Tokioka, and N. Nakagawa, Appl. Phys. Lett. 89, 062101 (2006).

${ }^{20}$ S. Tsurekawa, K. Kido, and T. Watanabe, Mater. Sci. Eng., A 462, 61 (2007).

${ }^{21}$ M. Kohyama and R. Yamamoto, Phys. Rev. B 50, 8502 (1994).

${ }^{22}$ R. Ishihara, T. M. He, V. Rana, Y. Hiroshima, S. Inoue, T. Shimoda, J. W. Metselaar, and C. I. M. Beenakker, Thin Solid Films 487, 97 (2005).

${ }^{23}$ M. Kohyama, Phys. Rev. B 50, 8502 (1994). 